\title{
EFEITOS DO TREINAMENTO MUSCULAR ESQUELÉTICO EM PACIENTES SUBMETIDOS À VENTILAÇÃO MECÂNICA PROLONGADA
}

Aenir Irena Borges Pedroso ${ }^{1}$, Marciéli Bigolin ${ }^{1}$, Marisa Pereira Gonçalves ${ }^{2}$, Roberta Weber Werle ${ }^{3}$

\begin{abstract}
RESUMO: Estudo com o objetivo de relatar os efeitos da aplicação do treinamento muscular esquelético em dois pacientes internados em unidade de terapia intensiva. Foram realizadas avaliações na internação; no desmame do ventilador mecânico; na alta da unidade e na alta hospitalar, tendo como parâmetros a avaliação nutricional e músculo-esquelética. O programa de treinamento iniciou na internação na unidade de terapia intensiva. Após a avaliação inicial, foram aplicados exercícios passivos até o período de desmame da ventilação. A partir desse período até a alta hospitalar foram aplicados exercícios ativos, testes de força muscular e goniometria. Houve melhora da força muscular e amplitude de movimento articular e o peso não sofreu redução importante, apesar da desnutrição encontrada em ambos os casos. O estudo demonstrou a importância do treinamento muscular em pacientes críticos, para minimizar os distúrbios músculo-esqueléticos devido ao repouso prolongado.
\end{abstract}

PALAVRAS-CHAVE: Unidades de terapia intensiva; Desnutrição proteica; Respiração artificial.

\section{EFFECTS OF THE SKELETAL MUSCLE TRAINING WITH PROLONGED MECHANICAL VENTILATION PATIENTS}

\begin{abstract}
The study aimed to verify the effects of a skeletal muscle training with intensive care unit (ICU) patients. It is a case report of two patients who underwent a program of skeletal muscle training. Nutritional and skeletal muscle assessments were performed during the hospital period, considering the period of weaning from mechanical ventilator, and ICU and hospital discharges. The training program started at the admission in ICU. After the evaluation of the patients, passive exercises were performed until the period of weaning. From weaning period until the hospital discharge, active exercises, tests of muscle strength and goniometry were conducted. As results, we found improvement in muscle strength and width of movement with the training. Weight, despite of the malnutrition found in both cases, did not suffer significant reduction. The study showed the importance of muscle training with critically ill patients to minimize skeletal muscle disorders due to prolonged bed rest.
\end{abstract}

KEYWORDS: Intensive care units; Protein malnutrition; Respiration, artificial.

\section{EFECTOS DELENTRENAMIENTO MUSCULAR ESQUELÉTICO EN PACIENTES SOMETIDOS A LA VENTILACIÓN MECÁNICA PROLONGADA}

RESUMEN: Estudio con el objetivo de relatar los efectos de la aplicación del entrenamiento muscular esquelético en dos pacientes internados en unidad de terapia intensiva. Fueron realizadas evaluaciones durante la internación; en el destete del ventilador mecánico; en el alta de la unidad y salida del hospital teniendo como parámetros la evaluación nutricional y músculo esquelético. El programa de entrenamiento empezó en la internación en la unidad de terapia intensiva. Después de la evaluación inicial, se aplicaron ejercicios pasivos hasta el periodo de destete de la ventilación. A partir de ese periodo hasta el alta hospitalaria fueron aplicados ejercicios activos, pruebas de fuerza muscular y goniometría. Hubo mejoría de la fuerza muscular y amplitud del movimiento articular y, el peso no sufrió reducción significativa, a pesar de la desnutrición encontrada en ambos casos. El estudio demostró la importancia del entrenamiento muscular, en pacientes críticos, para disminuir los disturbios músculo-esqueléticos debido al reposo prolongado.

PALABRAS CLAVE: Unidades de terapia intensiva; Desnutrición proteica; Respiración artificial.

\footnotetext{
${ }^{1}$ Fisioterapeuta graduada pela Universidade Federal de Santa Maria-UFSM.

${ }^{2}$ Fisioterapeuta. Doutor em Ciências da Saúde pela Universidade de Brasília-UnB. Docente do Departamento de Fisioterapia e Reabilitação da UFSM.

${ }^{3}$ Graduanda de Fisioterapia da UFSM.
}

Autor correspondente:

Roberta Weber Werle

Universidade Federal de Santa Maria

Rua Silva Jardim, 1341 - 97010-493 - Santa Maria-RS, Brasil

Recebido: 16/07/09

E-mail: robertawwerle@hotmail.com

Aprovado: 04/02/10

Cogitare Enferm 2010 Jan/Mar; 15(1):164-8 


\section{INTRODUÇÃO}

A Unidade de Terapia Intensiva (UTI) desempenha um papel crucial na sobrevida de pacientes gravemente enfermos, e tem o objetivo centrado na recuperação ou manutenção de suas funções fisiológicas. Neste contexto, diversas alterações secundárias podem manifestar-se, entre as quais se pode destacar a desnutrição e a disfunção muscular. A necessidade de suporte ventilatório mecânico comumente ocorre no paciente crítico devido a disfunções cardiopulmonares. Esse suporte, quando administrado por período prolongado, contribui para a imobilidade dos pacientes no leito.

O imobilismo reduz o glicogênio e adenosina trifosfato (ATP); a resistência muscular, que pode comprometer a irrigação sanguínea com consequente diminuição da capacidade oxidativa; a redução da força muscular e do torque, associados à falta de coordenação devido à fraqueza generalizada, contribuem para a atrofia das fibras musculares tipo I e II, o que resulta em má qualidade do movimento ${ }^{(1)} \mathrm{e}$ comprometimento do desmame da ventilação(2).

Doenças que promovem anormalidades metabólicas, como a síndrome de resposta inflamatória sistêmica, secundária à infecção, aceleram o processo de deficiência nutricional do paciente crítico ${ }^{(3)}$. Este resulta em diminuição da força máxima de movimentos voluntários, das pressões inspiratórias e expiratórias máximas, endurance muscular, resposta ventilatória à hipóxia e à hipercapnia e do ritmo de cicatrização de feridas $^{(4)}$. Ainda, imprime aumento da mortalidade, do tempo de internação e dos custos hospitalares ${ }^{(5)}$. Esta pesquisa objetivou demonstrar os efeitos de um programa de treinamento músculo-esquelético em pacientes submetidos à ventilação mecânica prolongada, tendo como parâmetros de análise a relação entre a avaliação nutricional e a musculatura esquelética.

\section{MÉTODO}

Relato de caso de dois pacientes adultos, internados na UTI de um hospital universitário submetidos à ventilação mecânica invasiva prolongada ${ }^{(6)}$, com estabilidade hemodinâmica e sem cardiopatia grave. O estudo foi aprovado por comitê de ética em pesquisa sob o nº 2008/53 e realizado com autorização dos responsáveis legais, mediante assinatura do Termo de Consentimento Livre e Esclarecido.

No período de agosto a dezembro de 2008, foi aplicado nos dois pacientes um programa de treinamento muscular esquelético diário, com duração de 30 minutos. Foram submetidos a avaliação nutricional, testes de força da musculatura esquelética e goniometria.

A avaliação nutricional constou de parâmetros antropométricos como peso, altura e índice de massa corporal (IMC). Quando da internação na UTI, o peso foi mensurado através da equação de estimativa de peso; a altura foi avaliada pela estatura recumbente, método alternativo realizado com o indivíduo em posição supina e com o leito em posição horizontal completa $^{(7)}$. O IMC foi calculado pela fórmula: peso atual $(\mathrm{Kg}) /$ estatura $(\mathrm{m})^{2}$.

No período entre o desmame e a alta hospitalar foram mensurados o peso, a altura, o IMC e a circunferência do braço (CB), e realizados testes de força muscular (bíceps braquial, tríceps braquial, deltóide, quadríceps femoral, flexores plantares, tibial anterior e posterior) e goniometria das principais articulações.

O treinamento muscular esquelético foi realizado observando diferentes modalidades de exercícios, dependendo da fase em que se encontravam os sujeitos da pesquisa:

Fase 1 - período entre a internação e fase de desmame. Exercícios passivos nas articulações dos membros superiores e inferiores, pelo tempo de 30 minutos, com séries de 10 repetições. Exercícios de flexão e extensão de ombro; pronação e supinação de antebraço; flexão palmar; dorsiflexão, desvio radial e ulnar de punho; arqueamento e achatamento do arco da mão; flexão e extensão, adução e abdução das articulações do polegar e demais dedos; abdução e adução do quadril; inversão e eversão do tornozelo; flexão e extensão, abdução e adução dos artelhos.

Fase 2 - período entre o fim do desmame e a alta hospitalar. Exercícios passivos, ativo-assistidos, ativos e ativos resistidos, conforme a evolução do paciente. Cada sessão teve uma duração de 30 minutos e foram feitas séries de 10 repetições para cada exercício.

\section{RELATO DOS CASOS E DISCUSSÃO}

Caso 1: paciente feminina, 22 anos, gestante de 20 semanas, $1^{\varrho}$ pós-operatório de laparotomia com diagnóstico de apendicite e sepse abdominal. A avaliação fisioterapêutica foi realizada no primeiro, nono, décimo primeiro e décimo segundo dias de internação. No 
segundo dia, a paciente apresentou aborto espontâneo. Após onze dias na UTI, teve alta da unidade e, no décimo segundo dia, alta hospitalar. Houve decréscimo do peso, do IMC e da CB entre a fase de internação na UTI e desmame; entretanto, estas variáveis mantiveramse constantes nas demais etapas (Tabela 1). Não houve mudança nos graus de força muscular (Tabela 2) e na goniometria das principais articulações, entre o desmame e a alta hospitalar (Tabela 3).

Caso 2: paciente feminina, 53 anos, diabética, com insuficiência renal crônica, colite pseudomembranosa, pneumonia aspirativa e parada cardio-respiratória na internação. Permaneceu 18 dias na UTI, tendo alta no décimo nono dia, e alta hospitalar após 45 dias de internação. Houve decréscimo no peso e no IMC entre a internação e a alta da UTI, e aumento entre a alta da UTI e a alta hospitalar A CB manteve-se constante entre a internação e o desmame, aumentou entre o desmame e a alta da UTI e diminuiu até a alta hospitalar (Tabela 1).

Tabela 1 - Descrição dos resultados do programa de treinamento músculo-esquelético desde a internação até a alta hospitalar.

\begin{tabular}{lcccccccc}
\hline & \multicolumn{2}{c}{ Dia } & \multicolumn{2}{c}{ Peso } & \multicolumn{2}{c}{ IMC * $^{*}$ CB* } \\
\hline Etapas & Caso 1 & Caso 2 & Caso 1 & Caso 2 & Caso 1 & Caso 2 & Caso 1 & Caso 2 \\
Internação na UTI & $1^{\circ}$ & $1^{\circ}$ & 60,5 & 58,5 & 25,21 & 21,99 & 31 & 27,5 \\
Desmame & $9^{\circ}$ & $10^{\circ}$ & 49,28 & 55,94 & 20,53 & 21,03 & 25 & 27,5 \\
Alta UTI & $11^{\circ}$ & $19^{\circ}$ & 49,28 & 52,29 & 20,53 & 19,66 & 25 & 28 \\
Alta Hospitalar & $12^{\circ}$ & $45^{\circ}$ & 49,28 & 54,5 & 20,53 & 20,49 & 25 & 25 \\
\hline
\end{tabular}

*IMC- índice de massa corporal. *CB- circunferência do braço.

A desnutrição energético-proteica é muito frequente em pacientes críticos, ocasionada pelo aporte nutricional inadequado e pela resposta metabólica ao estresse, levando ao hipermetabolismo e hipercatabolismo. A perda da massa magra eleva o risco de infecção e de mortalidade, retarda o desmame e pode ser letal quando se aproxima de $40 \%{ }^{(8-9)}$. O processo de desnutrição é agravado no decurso da internação, podendo evoluir para o quadro de hipermetabolismo, precedendo a Síndrome de Disfunção de Múltiplos Órgãos, responsável por até 85\% dos óbitos em UTI ${ }^{(10)}$.

Neste estudo, os dois pacientes apresentaram a sepse como complicação do quadro clínico. Adicionalmente, foi observada diminuição do peso corpóreo e do IMC. As infecções agudas aceleram a síntese e o catabolismo das proteínas corpóreas. A proteína muscular sofre proteólise, sendo rapidamente mobilizada para atender as necessidades de defesa orgânica na síntese de proteínas de fase aguda, como a proteína $\mathrm{C}$ reativa, anticorpos, imunoglobulinas, entre outros. Além disso, o balanço nitrogenado na sepse mostra-se negativo, observando que os processos catabólicos e a utilização dos aminoácidos para produção de energia contribuem substancialmente para este fato. Parte do balanço negativo se deve à anorexia, no entanto, a perda nitrogenada diária de um adulto com sepse situase entre 20g/dia a 35g/dia, correspondente à perda de 1
Kg de massa magra por dia ${ }^{(3)}$.

A desnutrição provoca uma redução no número de fibras, além de retardar a diferenciação morfológica, metabólica e contrátil característica dos tipos de fibra do músculo esquelético de ratos, durante o crescimento, ressaltando a importância da boa nutrição para evitar que os efeitos deletérios se tornem irreversíveis ${ }^{(11)}$.

Tabela 2 - Grau de força muscular no desmame e alta dos sujeitos hospitalizados.

\begin{tabular}{lcccc}
\hline & \multicolumn{2}{c}{ Desmame } & \multicolumn{2}{c}{ Alta hospitalar } \\
Músculos & Caso 1 & Caso 2 & Caso 1 & Caso 2 \\
\hline Bíceps Braquial & 3 & 3 & 3 & 4 \\
Tríceps Braquial & 4 & 3 & 4 & 4 \\
Deltoide & 3 & 3 & 3 & 3 \\
Quadríceps Femoral & 3 & 3 & 3 & 4 \\
Flexores Plantares & 3 & 2 & 3 & 3 \\
Tibial Anterior & 2 & 2 & 2 & 3 \\
Tibial Posterior & 3 & 2 & 3 & 3 \\
\hline
\end{tabular}

A CB diminuiu no caso 1 somente no período compreendido entre a internação e o desmame; ao contrário à do caso 2 que aumentou na alta da UTI e diminuiu na alta hospitalar. No caso 2, o fato da circunferência ter se mantido constante entre a internação e o desmame pode ser explicado pelo 
edema decorrente do acúmulo de líquido provocado pela insuficiência renal crônica.

O acúmulo de líquidos, que ocorre nos portadores de doença renal crônica, torna menos sensível o IMC para detectar desnutrição e tem menores reflexos sobre a medida da $\mathrm{CB}^{(12)}$. Isso explicaria o fato de não terem ocorrido grandes variações na medida da CB durante todas as etapas da hospitalização.

A força muscular aumentou entre o desmame e a alta hospitalar, nos dois casos. Evidências indicam que, alguns dias após o início de um programa de treinamento, ocorre melhora no desempenho muscular e no metabolismo, resultante de uma modificação no controle neuromuscular e/ou cardiovascular, o que melhora o desempenho das fibras musculares, o metabolismo e a distribuição do fluxo sanguíneo ${ }^{(3,13)}$.

Para cada semana de imobilização no leito, um paciente pode perder de 10 a $20 \%$ de seu nível inicial de força muscular, e em quatro semanas, $50 \%$ da força inicial pode estar perdida ${ }^{(1)}$. Um estudo demonstrou que uma semana de imobilização é suficiente para promover importantes alterações na morfometria e mecânica dos músculos sóleo e gastrocnêmio ${ }^{(14)}$.

Tabela 3 - Resultados da goniometria das principais articulações dos MMSS* e MMII** esquerdo e direito nas etapas de desmame e alta hospitalar.

\begin{tabular}{lcccccccc}
\hline & \multicolumn{3}{c}{ Desmame } & \multicolumn{5}{c}{ Alta hospitalar } \\
Goniometria & \multicolumn{2}{c}{ Caso 1 } & \multicolumn{2}{c}{ Caso 2 } & \multicolumn{2}{c}{ Caso 1 } & Caso 2 \\
& $\mathrm{E}$ & $\mathrm{D}$ & $\mathrm{E}$ & $\mathrm{D}$ & $\mathrm{E}$ & $\mathrm{D}$ & $\mathrm{E}$ & $\mathrm{D}$ \\
\hline Ombro & & & & & & & & \\
Flexão & $160^{\circ}$ & $165^{\circ}$ & $160^{\circ}$ & $160^{\circ}$ & $160^{\circ}$ & $165^{\circ}$ & $170^{\circ}$ & $170^{\circ}$ \\
Extensão & $10^{\circ}$ & $10^{\circ}$ & $10^{\circ}$ & $10^{\circ}$ & $10^{\circ}$ & $10^{\circ}$ & $15^{\circ}$ & $20^{\circ}$ \\
Cotovelo & & & & & & & & \\
Flexão-extensão & $140^{\circ}$ & $140^{\circ}$ & $100^{\circ}$ & $100^{\circ}$ & $140^{\circ}$ & $140^{\circ}$ & $110^{\circ}$ & $110^{\circ}$ \\
Quadril & & & & & & & & \\
Flexão & $90^{\circ}$ & $95^{\circ}$ & $90^{\circ}$ & $95^{\circ}$ & $90^{\circ}$ & $95^{\circ}$ & $100^{\circ}$ & $105^{\circ}$ \\
Extensão & $0^{\circ}$ & $0^{\circ}$ & $0^{\circ}$ & $0^{\circ}$ & $0^{\circ}$ & $00^{\circ}$ & $5^{\circ}$ & $5^{\circ}$ \\
Joelho & & & & & & & & \\
Flexão & $135^{\circ}$ & $135^{\circ}$ & $130^{\circ}$ & $130^{\circ}$ & $135^{\circ}$ & $135^{\circ}$ & $130^{\circ}$ & $130^{\circ}$ \\
Tornozelo & & & & & & & & \\
Dorsiflexão & $10^{\circ}$ & $10^{\circ}$ & $10^{\circ}$ & $10^{\circ}$ & $10^{\circ}$ & $10^{\circ}$ & $10^{\circ}$ & $10^{\circ}$ \\
Plantiflexão & $30^{\circ}$ & $35^{\circ}$ & $20^{\circ}$ & $20^{\circ}$ & $30^{\circ}$ & $35^{\circ}$ & $20^{\circ}$ & $20^{\circ}$ \\
\hline
\end{tabular}

*MMSS - Membros Superiores. **MMII - Membros Inferiores

Com o objetivo de manter a mobilidade das articulações e dos tecidos moles, de modo a minimizar a perda de flexibilidade dos tecidos e a formação de contraturas, foram administrados exercícios de amplitude de movimento ${ }^{(7)}$. Muitos fatores podem levar a uma amplitude de movimento reduzida, como doenças sistêmicas, articulares, neurológicas e musculares; lesões cirúrgicas ou traumáticas; ou apenas inatividade ou imobilidade por qualquer razão. Neste estudo, observouse aumento da amplitude de movimento entre o desmame e a alta hospitalar, nos dois casos, o que demonstrou a reversibilidade da condição do imobilismo traduzido na melhora da capacidade funcional desses sujeitos.

\section{CONCLUSÃO}

O presente estudo demonstrou a importância do treinamento muscular em pacientes críticos, para minimizar os distúrbios músculo-esqueléticos, devido ao repouso prolongado. O treinamento muscular promoveu melhora na amplitude de movimento articular e na força muscular, minimizando os efeitos negativos do imobilismo e prevenindo as limitações funcionais ${ }^{(15)}$.

Os resultados encontrados podem estimular a realização de novas pesquisas com treinamento muscular precoce em pacientes críticos, com o objetivo de diminuir os efeitos negativos da imobilização prolongada ${ }^{(16)}$. 
Desta forma, os resultados provenientes desta investigação e de outras que dela decorrerem poderão oferecer contribuições úteis no planejamento de medidas, visando à integralidade da assistência ao doente em unidades de terapia intensiva.

\section{REFERÊNCIAS}

1. Melo ACR, Lopez RFA. Efeitos da atividade física na redução dos efeitos do imobilismo. Estudo de caso. E F Deportes [periódico na Internet]. 2004; 10(72). [acesso em 2008 Out 15]. Disponível: http://www.efdeportes.com /efd72/imobil.htm

2. Machado CD. Fisioterapia hospitalar: efeitos fisiológicos da imobilização. Fisioweb 2002 [acesso em 2008 Out 15]. Disponível: http://www.wgate.com.br/conteudo/ medicinaesaude/fisioterapia/variedades/efeitos_imob.htm

3. Feferbaum R. Alterações metabólicas durante a sepse na criança. IMeN. [periódico na Internet]. 2005 [acesso 2008 Set 23]. Disponível: http://www.nutricaoclinica.com.br/

4. Associação de Medicina Intensiva Brasileira. Terapia nutricional no paciente grave. Rio de Janeiro: Revinter; 2001.

5. Pichard C, Kyle UG, Morabia A, Perrier A, Vermeulen B, Unger P. Nutritional assesment: lean body mass depletion at hospital admission is associated with an increased lenght of stay. Am J Clin Nutr. 2004;79:613-8

6. Amato MBP, coordenador. III Consenso de Ventilação Mecânica. J Pneumol. 2007;33(2):119-27

7. Cuppari, L. Nutrição clínica do adulto. São Paulo(SP): Manole; 2002

8. Kisner C, Colby, LA. Exercícios terapêuticos: fundamentos e técnicas. $4^{\mathrm{a}}$ ed. São Paulo(SP): Manole; 2005

9. Boles JM, Bion J, Connors A, Herridge M, Marsh B, Melot C. Weaning from mechanical ventilation. Eur Respir J. 2007;29:1033-56

10. Minicucci MF. Terapia nutricional no paciente crítico: o papel dos macronutrientes. Nutrire. 2006;31(1):97-109

11. Alves AP, Dâmaso AR, Pai VD. Efeito da desnutrição protéica pré e pós-natal sobre a morfologia, a diferenciação e o metabolismo do tecido muscular estriado esquelético em ratos. J Ped. 2008; 84(3):264-71

12. Santos PR, Coelho ML, Gomes NP, Josué CEP. Associação de indicadores nutricionais com qualidade de vida em pacientes portadores de doença renal crônica em hemodiálise. J Bras Nefrol. 2006;28(2):57-64

13. Melo ACR, Lopez RFA. Efeitos da imobilização prolongada e atividade física. E F Deportes [periódico na Internet]. 2002;8(50). [acesso em 2008 Out 12]. Disponível: http:// www.efdeportes.com/efd50/efeitos.htm

14. Lima SC. Curto período de imobilização provoca alterações morfométricas e mecânicas no músculo de rato. Rev Bras Fisioter. 2007;11(4):297-302

15. Cyrino ES, Oliveira AR, Leite JC, Porto DB, Dias RMR, Segantin AQ. Comportamento de flexibilidade após 10 semanas de treinamento com peso. Rev Bras Med Esporte. 2004; 10(4):87-99.

16. Porta R, Vitacca M, Gilè LS, Clini E, Bianchi L, Zanotti E. Supported arm training in patients recently weaned from mechanical ventilation. Chest. 2005; 128:2511- 20.

Cogitare Enferm 2010 Jan/Mar; 15(1):164-8 